\title{
1 Sex-biased reduction in reproductive success drives selective constraint on 2 human genes \\ 3
}

4 Eugene J. Gardner ${ }^{1}$, Matthew D. C. Neville ${ }^{1}$, Kaitlin E. Samocha ${ }^{1}$, Kieron Barclay ${ }^{2,3,4}$, Martin

5 Kolk $^{3}$, Mari E. K. Niemi ${ }^{1}$, George Kirov ${ }^{5}$, Hilary C. Martin ${ }^{1}$, Matthew E. Hurles ${ }^{1, *}$

6

$7{ }^{1}$ Wellcome Sanger Institute, Wellcome Genome Campus, Cambridge, Hinxton, United

8 Kingdom

9 'Max Planck Institute for Demographic Research, Rostock, Germany

$10{ }^{3}$ Demography Unit, Department of Sociology, Stockholm University, Stockholm, Sweden

$11{ }^{4}$ Swedish Collegium for Advanced Study, Uppsala, Sweden

$12{ }^{5}$ Division of Psychological Medicine and Clinical Neurosciences, School of Medicine, Cardiff

13 University, Cardiff, UK

14 *meh@sanger.ac.uk

\section{${ }_{15}$ Summary}

16 Genome-wide sequencing of human populations has revealed substantial variation among

17 genes in the intensity of purifying selection acting on damaging genetic variants. While

18 genes under the strongest selective constraint are highly enriched for Mendelian disorders,

19 most of these genes are not associated with disease and therefore the nature of the

20 selection acting on them is not known. Here we show that genetic variants that damage

21 these genes reduce reproductive success substantially in males but much less so in

22 females. We present evidence that this reduction is mediated primarily by cognitive and

23 behavioural traits, which renders male carriers of such variants less likely to find mating

24 partners. These findings represent strong genetic evidence that sexual selection mediated

25 through female mate choice is shaping the gene pool of contemporary human populations.

26 Furthermore, these results suggest that sexual selection accounts for $21 \%$ of purifying

27 selection against heterozygous variants that ablate protein-coding genes.

\section{Main text}

29 The most damaging genetic variants, gene deletions and protein-truncating variants (PTVs),

30 are removed from the population by selection with strength that varies substantially from

31 gene to gene ${ }^{1,2}$. The strength of selection against heterozygous PTVs has been estimated by

32 the selection coefficient, $\mathbf{s}_{\text {het }}$, which exhibits a continuous spectrum across human genes ${ }^{3,4}$,

33 although most attention has been focused on a subset of $\sim 3,000$ genes with the highest

34 probability of Loss-of-function Intolerance $(\mathrm{pLI})^{1}$.

36 The selection pressures acting on these most selectively constrained genes have not been

37 fully characterised, but, a priori, could include natural selection against variants increasing

38 pre-reproductive mortality or decreasing fertility, and sexual selection acting on mate choice

39 or intra-sexual competition ${ }^{5}$. Gene deletions and PTVs in these genes have been shown to

40 be associated with lower educational attainment ${ }^{6,7}$ and general intelligence ${ }^{8}$, as well as

41 increased risk of intellectual disability, and some psychiatric disorders ${ }^{9}$. Moreover, these 
constrained genes are strongly enriched for dominant early-onset Mendelian diseases (including many neurodevelopmental disorders), many of which are associated with increased pre-reproductive mortality, indicating that natural selection likely makes a substantive contribution to this selective constraint. However, the majority $(65 \%)$ of constrained genes $(\mathrm{pLI}>0.9)$ have not yet been associated with a Mendelian disease. This raises the fundamental question of whether natural selection represents the sole mechanism imposing this form of selective constraint on human genes, or whether other forms of selection are at work.

To explore the nature of selection acting on these genes we identified PTVs and genic deletions in the UK Biobank ${ }^{10}$ comprising largely post-reproductive individuals (median age at recruitment: 58 years, range: 39-73 years, birth years: 1934-1970; Supplementary Figure 1 ), and investigated the association with reproductive success. We called large copy number variants (deletions and duplications) from SNP genotyping array data on 340,925 unrelated participants of European ancestry (Supplementary Figure 2), and identified PTVs from exome sequencing among a subset of 34,812 participants (Supplementary Figure 3$)^{11}$. For each participant, we then calculated the cumulative burden of private (only observed in one individual), heterozygous genic deletions and PTVs by combining $s_{\text {het }}$ selection coefficients of each autosomal gene impacted by these variants (under the assumption that fitness is multiplicative, see Methods), which we term their $s_{\text {het }}$ burden. The distribution of $s_{\text {het }}$ burden was statistically indistinguishable between males and females: for participants with only genic deletion data available, $0.56 \%$ and $0.55 \%$ respectively had an $\mathrm{s}_{\text {het }}$ burden $\geq 0.15$ (Fisher's $p=0.61$; Figure 1B), and for participants with both genic deletion and PTV data available the analogous proportions were $6.99 \%$ and $7.06 \%$ (Fisher's $p=0.80$; Figure $1 C$ ).

We assessed the relationship between $s_{\text {het }}$ burden and number of children, using a linear regression correcting for age and population structure (Methods; Supplementary Figure 4; Supplementary Table 1). We observed that an $\mathrm{s}_{\text {net }}$ burden of 1 is associated with a decrease in the overall total number of overall children for both males $(0.53$ fewer children $[95 \% \mathrm{Cl}$ $\left.0.36-0.71], p=2.2 \times 10^{-9}\right)$ and females $(0.17$ fewer children [95\% $\left.\mathrm{Cl} 0.01-0.33], \mathrm{p}=0.04\right)$ when combining results from deletion and PTV-based analyses.

To determine if the observed effect of $s_{\text {het }}$ burden was due to an actual reduction in overall number of children or a result of selection against having children at all, we performed two analyses. Firstly, we evaluated childlessness using logistic regression and again observed a striking sex difference in participants' probability of having any children given their $\mathrm{s}_{\text {het }}$ burden, for both PTVs and genic deletions (Figure 1A). Combining the analyses of genic deletions and PTVs, we found that an $\mathrm{s}_{\text {het }}$ burden of 1 decreases the probability of males having any children $\left(\mathrm{OR}=0.29[95 \% \mathrm{Cl} 0.21-0.40], \mathrm{p}=9.0 \times 10^{-14}\right)$ much more than females $(\mathrm{OR}=0.69[95 \% \mathrm{Cl} 0.49-0.98], \mathrm{p}=0.04)$. We also observed that private duplications and likely damaging private missense variants exhibit similar but weaker effects on childlessness (Supplementary Figure 5). Secondly, if we remove childless individuals from the analysis, $\mathbf{s}_{\text {het }}$ burden ceases to have a significant effect on the number of offspring, confirming that the observed decrease in reproductive success is determined largely by increased childlessness (Supplementary Figure 6). 
88 We also considered whether ascertainment biases or differences in fertility between the UK

89 Biobank population and the UK population as a whole could affect these results. As UK

90 Biobank participants included in these analyses are biased towards females (54\%), the

91 observed sex bias is not due to having greater statistical power to detect an effect on

92 reproductive success in males. Likewise, fertility rates between the UK Biobank population

93 and the UK population as a whole are highly similar; the average total fertility rate in the UK

94 from 1983-2000 (where data are available for both males and females), when UK Biobank

95 participants would have been reproductively active, was 1.78 for males ${ }^{12}$ and 1.76 for

96 females $^{13}$, which is very similar to that observed among UK Biobank participants (average

97 number of children for males $=1.77$; females $=1.80$ ).

99 We observed consistent sex-bias in the association of $\mathbf{s}_{\text {het }}$ burden with childlessness when

100 performing this analysis in different ways, including: (i) limiting analyses to carriers of private

101 genic deletions or PTVs in the genes under most selective constraint (following thresholds

102 set by their authors: $p L I \geq 0.9$ or $s_{\text {het }} \geq 0.15$; Supplementary Figure 7 ), (ii) extending analysis

103 to more frequent, but still rare genic deletions and PTVs (Supplementary Figure 8), (iii)

104 excluding genes known to cause Mendelian disorders (male OR=0.32 [95\% Cl 0.21-0.47],

$105 \mathrm{p}=2.1 \times 10^{-8}$ ), and (iv) restricting analysis to individuals in specific age ranges (Supplementary

106 Figure 9). 
A
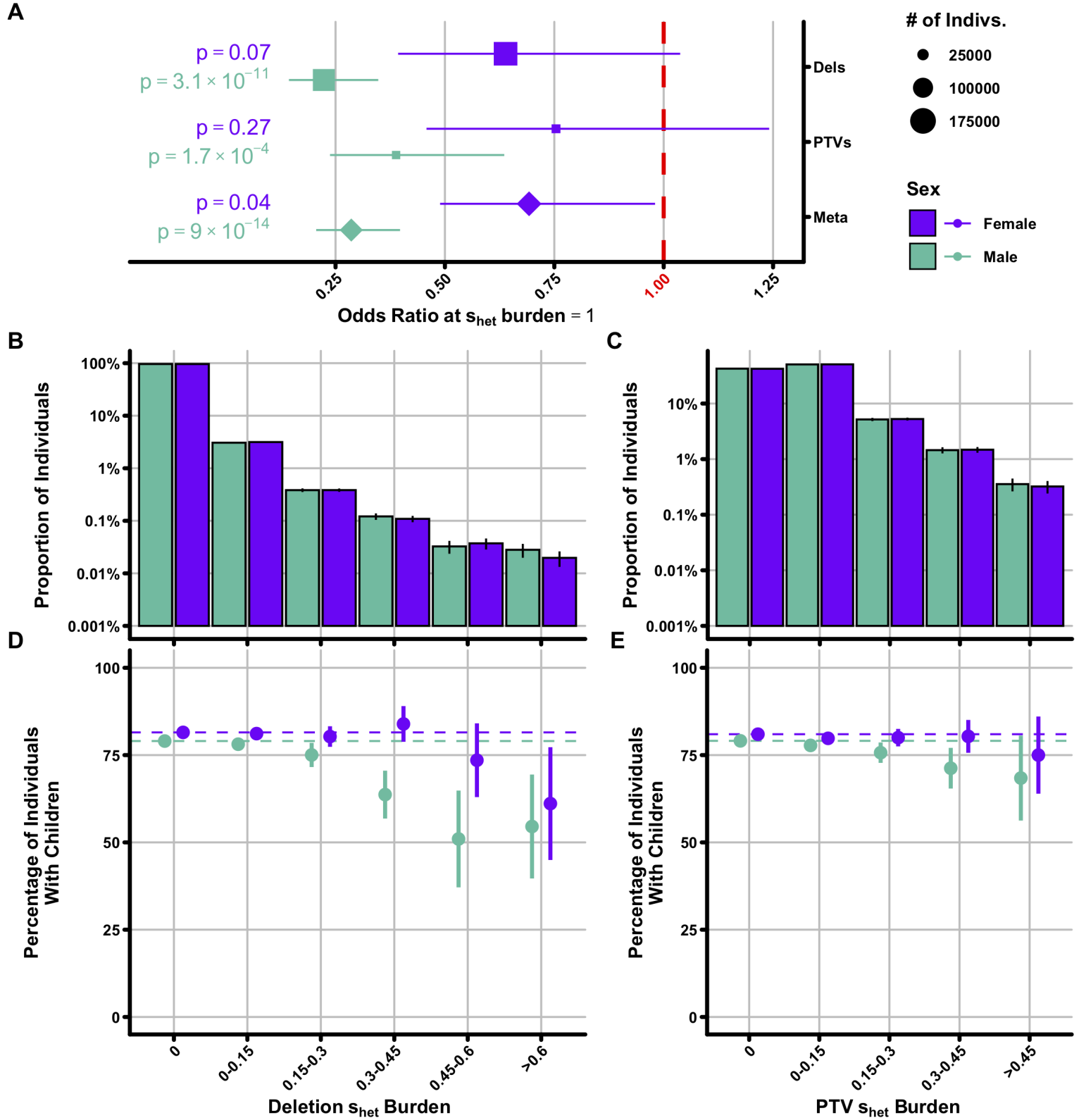

108 Figure 1. Differences in male and female reproductive success as a function of cumulative rare

\section{9 deleterious genetic variation.}

110 (A) Odds ratio estimates for the effect of cumulative deleterious variation for deletions, PTVs, and a

111 combined meta-analysis on childlessness separated for males (jade) and females (violet). Number of

112 individuals included in each analysis is indicated by the size of the point. (B; C) Proportion of

113 individuals in $0.15 s_{\text {het }}$ bins for deletions (B) and PTVs (C). (D; E) Percentage of individuals with children in bins based on $s_{\text {het }}$ burden for deletions (D) and PTVs (E). All error bars are $95 \%$ confidence intervals calculated on the population proportion.

117 We evaluated three hypotheses that could account for increased childlessness: (i) impaired

118 fertility (e.g. inability to produce viable gametes), (ii) adverse health conditions, and (iii)

119 cognitive and behavioural factors (which could decrease ability to find a mate, or increase

120 voluntary childlessness). We observed that $s_{\text {het }}$ burden does increase the risk of male

121 infertility, albeit with wide confidence intervals, $(O R=8.93$ [95\% Cl 1.55-51.51], $p=0.01)$ but

122 not female infertility $(O R=0.54$ [95\% Cl $0.17-1.75], p=0.31)$; however, three lines of evidence 
suggest impaired fertility is not the predominant cause of the sex-biased association between $s_{\text {het }}$ burden and childlessness. First, when introducing male infertility status available from combined health outcomes data for all UK Biobank participants (combined hospital episode statistics, primary care records, self-reported conditions and death records) as a covariate in the association testing, we observed minimal reduction in the strength of the association between $s_{\text {het }}$ burden and male childlessness (OR=0.29 [95\% $\mathrm{Cl} 0.21-0.40$ ], $p=1.5 \times 10^{-13}$; Supplementary Table 2). Second, we observed minimal change in the association between $s_{\text {het }}$ burden and male reproductive success after removing all 150 autosomal genes for which at least limited evidence exists of an association to male infertility $\left(\mathrm{OR}=0.29[95 \% \mathrm{Cl} 0.20-0.40], \mathrm{p}=1.0 \times 10^{-13}\right)^{14}$ or all 742 genes associated with male infertility in mice $\left(\mathrm{OR}=0.29[95 \% \mathrm{Cl} 0.20-0.40], \mathrm{p}=6.8 \times 10^{-13}\right)^{15}$. Finally, genes under the highest selective constraint $\left(s_{\text {het }} \geq 0.15\right)$ are not associated with higher expression levels in testis, unlike the genes currently known to be associated with male infertility (Supplementary Figure 10). Together, these findings are consistent with a previous study that sought but did not find a widespread role for highly-penetrant dominant deletions in spermatogenic failure ${ }^{16}$.

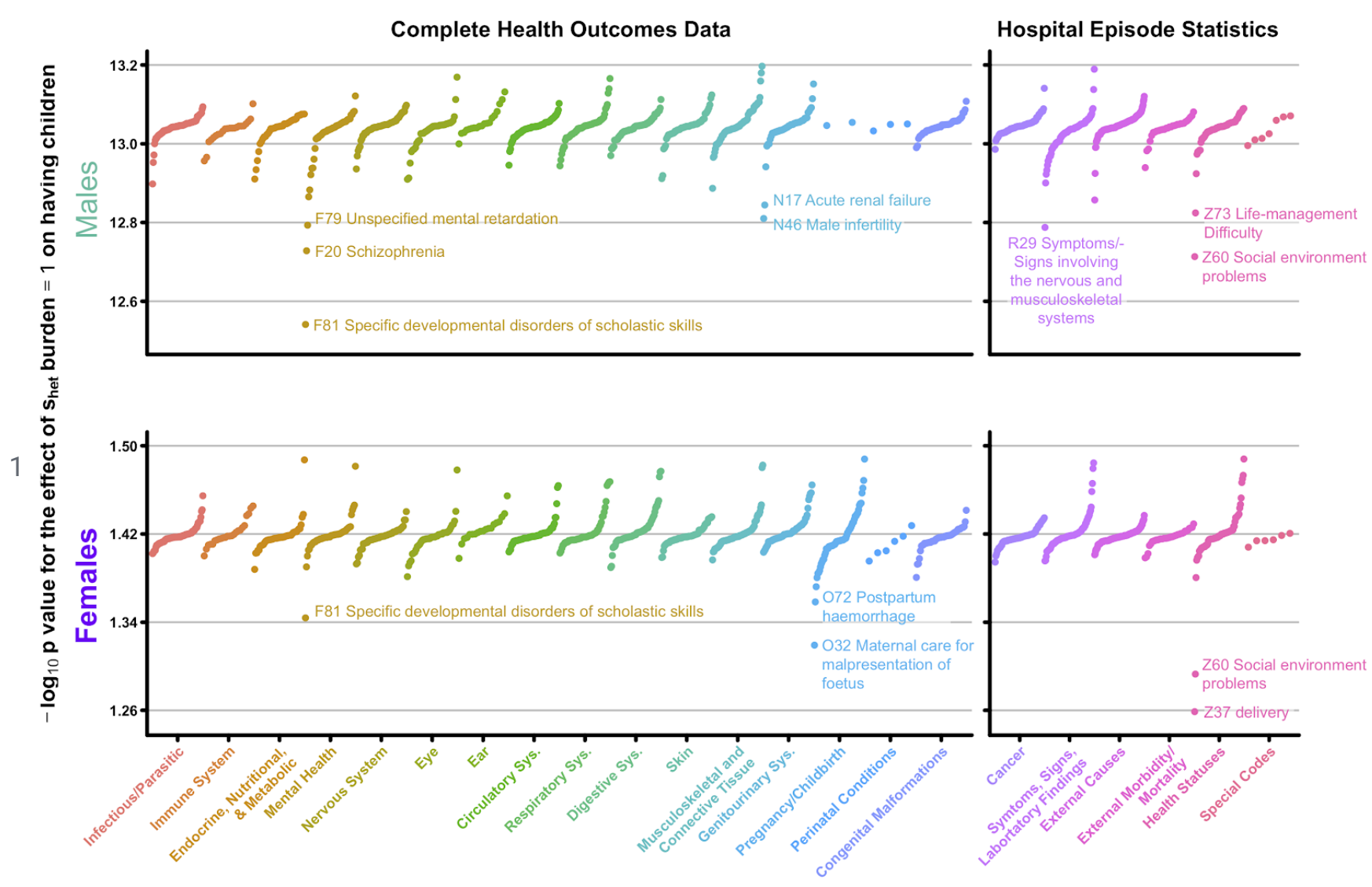

140 Figure 2. Modulation of the relationship between $\boldsymbol{s}_{\text {het }}$ burden and childlessness by various disorders.

142 Plotted is the deletion and PTV meta-analysis $-\log _{10} p$ value for the association between $s_{\text {het }}$ burden and having children, corrected by one of 1,294 ICD-10 codes from a combination of general

144 practitioner, hospital episode records, and self-reported conditions (left) or hospital episode records

145 alone (right) separately for males (top) and females (bottom). Results are ordered first by ICD-10 chapter ( $x$-axis) and then by increasing - $\log _{10} p$ value ( $y$-axis). Visual outliers are labelled. 
148 Previous studies have shown that a variety of physical birth defects are associated with

149 reduced reproductive success ${ }^{17,18}$. We comprehensively assessed whether any adverse

150 health conditions contributed to the association between $\mathbf{s}_{\text {het }}$ burden and childlessness. We

151 independently tested 19,154 ICD-10 codes (from both hospital episode statistics and

152 combined health outcomes data across four levels of the ICD-10 hierarchy; Methods) as a

153 covariate in the association test of $\mathbf{s}_{\text {het }}$ burden on childlessness (Figure 2; Supplementary

154 Figures 11-13; Supplementary Table 2; Methods). We found that while many ICD-10 codes

155 are associated with having children, in particular positive associations with male-specific

156 codes for elective sterilisation and female-specific codes associated with pregnancy and

157 childbirth (Supplementary Figure 11), correcting for any ICD-10 code had minimal impact on

158 the strength of association between $\mathbf{s}_{\text {het }}$ burden and childlessness (Figure 2; Supplementary

159 Figures 12,13$)$. The biggest impact on the association of $s_{\text {het }}$ burden and male childlessness

160 was observed for 'developmental disorders of scholastic skills', although this only modestly

161 reduced the significance of the association from $p=9.0 \times 10^{-14}$ to $p=2.9 \times 10^{-13}$ (Figure 2;

162 Supplementary Table 2). In addition to diseases, clinically annotated ICD-10 codes are also

163 available for a range of factors denoting health status and contact with health services

164 (Chapter XXI - Health Statuses). We noted that two of these also had a modest impact on

165 the association of $\mathbf{s}_{\text {het }}$ burden and male childlessness when included as covariates in

166 association testing (Figure 2; Supplementary Table 2). These were codes relating to life

167 management difficulty (code Z73) and social environment problems (code Z60), with the

168 latter driven primarily by the status of living alone (code Z60.2). Both of these two

169 health-related factors are positively associated with $\mathbf{s}_{\text {het }}$ burden.

171 There is substantial existing evidence that behavioural and cognitive traits influence

172 reproductive success in a sex-biased manner. First, the reduced reproductive success

173 associated with a range of psychiatric disorders is much more pronounced in males than in

174 females $^{19}$. Second, personality traits associated with increased reproductive success differ

175 between males and females, with increased extraversion in males but greater neuroticism in

176 females being linked to increased reproductive success ${ }^{20}$. Third, although the most highly

177 ranked mate characteristics are highly concordant between the sexes ${ }^{21}$, some mate

178 preferences differ between the sexes, with males placing greater value on physical

179 attractiveness and females valuing cues relating to earning potential ${ }^{20-23}$. Finally, low

180 socioeconomic status and low educational attainment have been more strongly linked to

181 increased childlessness in males than females across populations ${ }^{24-27}$. This has typically

182 been ascribed to males of lower socioeconomic status finding it harder to attract a

183 partner ${ }^{28,29}$.

185 Some of these observations about sex-biased reproductive success have been related to

186 sexual selection by mate choice, in which one sex (typically female) tends to be more

187 discriminating in their choice of mates. Alternative theories have been proposed regarding

188 the causes of sexual selection, including those that focus on disparities in gamete size

189 (Darwin-Bateman paradigm ${ }^{30}$ ) and others that focus on differential parental investment ${ }^{31}$.

190 This latter hypothesis posits that sexual selection by mate choice is driven, in large part, by

191 the sex that invests more in offspring (typically female) being more discriminating in their

192 choice of mates, especially with regard to their potential to invest in offspring. However, a

193 sex-biased reduction in reproductive success need not be caused by sex differences in mate 
194 preferences. Sex-biased reproductive success could also result from a sex bias in trait

195 severity coupled to mate choice preferences that are not sex-biased. These mechanisms are

196 not mutually exclusive; both could be contributing to an overall sex-biased reduction in

197 reproductive success, albeit on different traits.

199 A key prediction of the hypothesis that differential mate choice underpins the observation of

200 a male-biased association of $\mathbf{s}_{\text {het }}$ burden with increased childlessness is that males with a

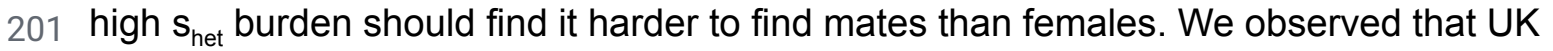

202 Biobank participants with high $\mathbf{s}_{\text {het }}$ burden were significantly less likely to have reported

203 currently living with a partner (at the time of assessment), consistent with the findings from

204 the ICD10 codes, and that, like reproductive success, this effect was significantly stronger in

205 males than in females (Figure 3A). UK Biobank males currently living with a partner are also

206 much more likely to have children (OR=5.80 [95\% Cl 5.65-5.96] , $p<1 \times 10^{-100}$;

207 Supplementary Figure 14). We note that the status of currently living with a partner is an

208 imperfect proxy for partner status during peak reproductive years, but the latter information is

209 not currently available in UK Biobank. We also found that $s_{\text {het }}$ burden was significantly

210 positively associated with reporting never having had sex for both male $(\mathrm{OR}=0.07[95 \% \mathrm{Cl}$

$\left.2110.03-0.20], p=4.3 \times 10^{-07}\right)$ and female (OR=0.18 [95\% Cl 0.05-0.61], $\left.p=5.8 \times 10^{-03}\right)$ UK Biobank

212 participants, without significant sex-bias (Figure 3B). Additionally, while same sex sexual

213 behaviour is strongly associated with increased childlessness in UK Biobank (male OR=0.14

214 [95\% Cl 0.13-0.15], $p<1 \times 10^{-100}$; female OR=0.27 [95\% Cl 0.25-0.29] $p<1 \times 10^{-100}$;

215 Supplementary Figure 14), we observed no significant impact of $\mathbf{s}_{\text {het }}$ burden on the likelihood

216 of having engaged in same sex sexual behaviour among males $(\mathrm{OR}=1.27[95 \% \mathrm{Cl}$

217 0.59-2.69], $p=0.54$; Supplementary Figure 15) nor did we observe any change in the

218 relationship between $\mathbf{s}_{\text {het }}$ burden and childlessness when excluding individuals who engaged

219 in same-sex sexual behaviour from our primary model $(\mathrm{OR}=0.27$ [95\% $\mathrm{Cl} 0.19-0.39]$,

$\left.220 \mathrm{p}=1.2 \times 10^{-13}\right)$. 


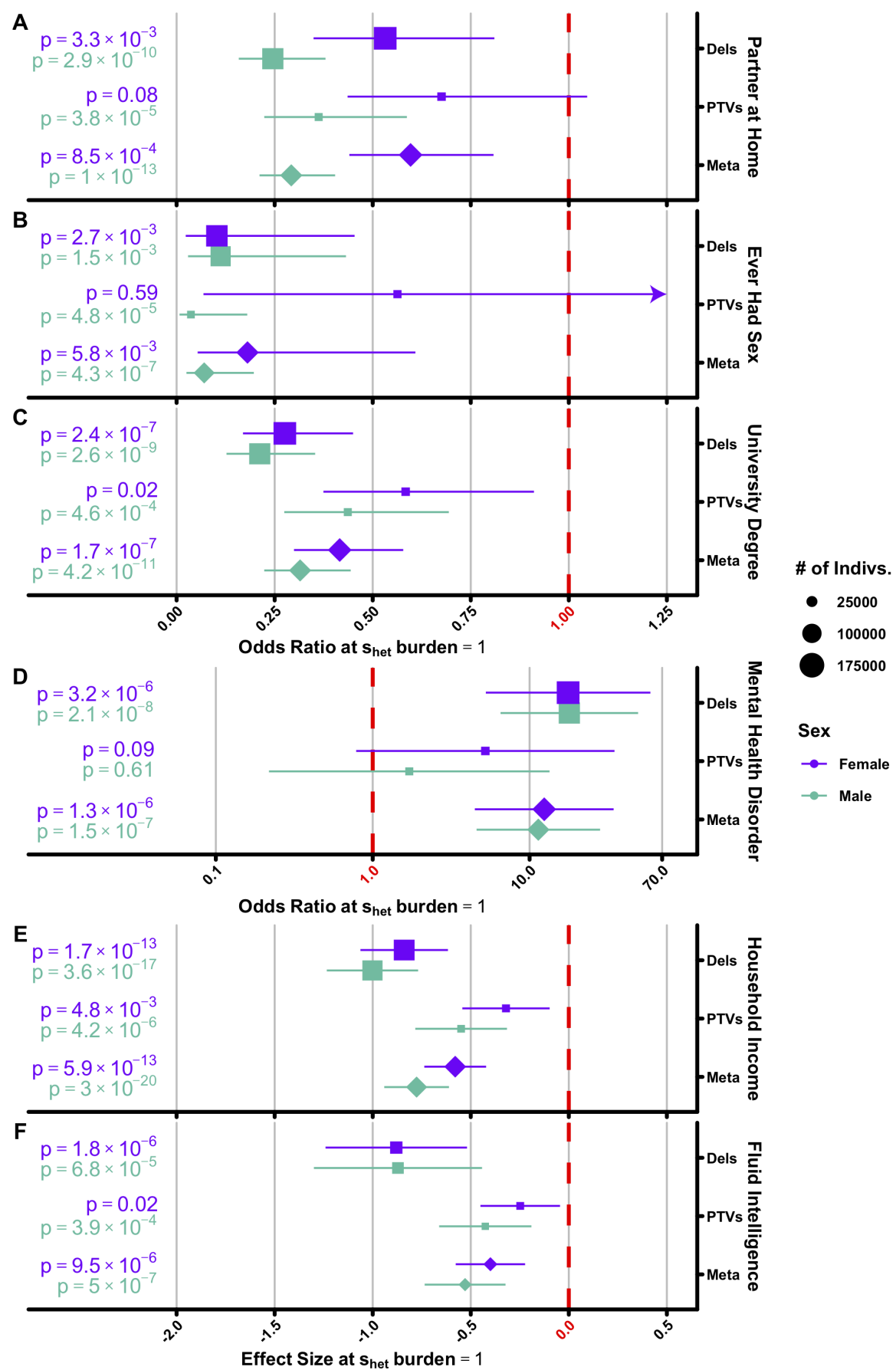

Figure 3. Effect of $s_{\text {het }}$ burden on traits known to be associated with reproductive success.

Shown are similar plots to Figure 1A, except for six phenotypes which have been previously associated with reproductive success: $(A)$ having a partner at home, $(B)$ ever having engaged in sexual intercourse, (C) educational attainment as measured by having a university degree, (D) household income (as measured by income bracket and corrected for having a partner at home; see methods), (E) fluid intelligence (in standard deviations), and ( $F$ ) having a mental health disorder. For each trait, we tested using a logistic $(A, B, C, D)$ or linear $(E, F)$ model the effect of $s_{\text {het }}$ burden on each phenotype shown above, corrected for age, age ${ }^{2}$, and the first thirty ancestry principal components.

230 The arrow in plot (B) indicates the upper confidence interval for female PTVs is outside the range of

231 the $X$-axis. Note that plot $(D)$ is in log rather than linear scale. 
233 We explored in UK Biobank whether the impact of $\mathbf{s}_{\text {het }}$ burden on reproductive success might

234 plausibly be mediated through some of the specific factors highlighted by the previous

235 psychiatric, demographic and psychosocial research summarised above. Firstly we

236 investigated the impact of $s_{\text {het }}$ burden on cognition as measured by fluid intelligence in

237 110,190 (51,378 males, 58,812 females) UK Biobank participants. We found that $\mathbf{s}_{\text {het }}$ burden

238 was associated with significantly reduced fluid intelligence scores of males and females with

239 similar effect sizes (Figure $3 F$ ). Increasing $s_{\text {het }}$ burden is also associated with lower

240 educational attainment and household income (Figure 3C,E), again with similar effect sizes

241 in males and females. To evaluate the potential impact of this reduced cognition on male

242 reproductive success, we extended previously published work relating the results of IQ tests

243 taken by $95 \%$ of Swedish males (during military conscription) to their completed family

244 size $^{32}$. We estimated that the decrement in cognition observed in UK Biobank males could

245 account for $6 \%$ [95\% Cl 5\%-9\%] of the reduced male reproductive success associated with

246 high $\mathrm{s}_{\text {het }}$ burden (Supplementary Figure 16, Methods). We also note that the decrease of

247 reproductive success with decreasing IQ was most pronounced in males with IQ $<70$

248 (Supplementary Figure 17) ${ }^{32}$, who are likely depleted in UK Biobank relative to the general

249 population.

251 Analysis of psychiatric disorders in UK Biobank is complicated by both recruitment bias away

252 from more severe psychiatric disorders and incomplete data on participants ${ }^{10,33-35}$. The most

253 comprehensive data are available on a subset of UK Biobank individuals from a mental health questionnaire for which participants were invited by email $(n=157,366)^{34}$. We observed that a high $\mathrm{s}_{\text {het }}$ burden was very strongly associated with not having an email address (male OR=0.30 [95\% Cl 0.21-0.41], $p=8.4 \times 10^{-14}$; female OR=0.48 [95\% Cl $0.35-0.65], p=3.1 \times 10^{-6}$; Supplementary Figure 18), which likely explains why individuals with a high $s_{\text {het }}$ burden were much less likely to complete the questionnaire (male OR $=0.43[95 \%$ Cl 0.31-0.60], $p=7.6 \times 10^{-7}$; female $\mathrm{OR}=0.41$ [95\% $\mathrm{Cl} 0.30-0.56$ ], $p=1.7 \times 10^{-8}$; Supplementary Figure 18). Therefore we focused analyses of mental health disorders on the complete health outcomes data available for all participants. These data corroborate a previous finding ${ }^{9}$ that high $\mathrm{s}_{\text {het }}$ burden increases the risk of psychiatric disorders previously associated with reduced reproductive success (intellectual disability, schizophrenia, autism, attention deficit hyperactive disorder, and bipolar disorder; Figure 3D, Supplementary Figure 19) ${ }^{19}$, and that these psychiatric disorders are associated with increased childlessness in both male $\left(\mathrm{OR}=0.35\right.$ [95\% Cl 0.32-0.39], $\left.\mathrm{p}=8.3 \times 10^{-85}\right)$ and female $(\mathrm{OR}=0.58$ [95\% Cl 0.52-0.65], $p=2.7 \times 10^{-19}$ ) UK Biobank participants, albeit with substantial sex-bias (Supplementary Figure 14). This finding accords with a previous study showing that copy number variants associated with increased risk of schizophrenia are also associated with disproportionately reduced reproductive success in males ${ }^{36}$. Carriers of well-characterised neurodevelopmental disorder-associated copy number variants, which include those with a strong association to schizophrenia (Methods), only account for $3.7 \%(n=12,608)$ of individuals in UK Biobank. Removal of these individuals from the dataset does not significantly alter the association of $\mathrm{S}_{\text {het }}$ burden with reduced male reproductive success (OR=0.29 [95\% Cl 0.21-0.41], $p=3.1 \times 10^{-12}$ ).

We subsequently tested the impact of $s_{\text {het }}$ burden on childlessness only for individuals without any evidence of a mental health disorder associated with reduced reproductive 
279 success (from hospital episode statistics, combined health outcomes data, or the mental

280 health questionnaire). We observed very similar effect sizes to when analysing all individuals

281 (male OR=0.31 [95\% Cl 0.22-0.43], $\mathrm{p}=1.0 \times 10^{-11}$; female OR=0.75 [95\% Cl 0.53-1.07],

$282 \mathrm{p}=0.12$ ), suggesting that the effect on childlessness is not predominantly driven by this

283 subset of mental health disorders. We explored this further, using data external to UK

284 Biobank that are less affected by the limitations described above. Using previous estimates

285 of the increased risk of mental health disorders caused by PTVs in highly constrained

286 genes $^{9}$, and the reduced reproductive success associated with those disorders ${ }^{19}$, we

287 estimated that these mental health disorders could account for $15 \%$ [ 7 - 33\%] of the reduced

288 male reproductive success associated with high $\mathrm{s}_{\text {het }}$ burden (Methods). Thus, in UK Biobank,

289 both reduced fluid intelligence and increased risk of psychiatric disorders account for only

290 modest proportions of increased male childlessness due to $\mathbf{s}_{\text {het }}$ burden.

292 We next used multiple regression to explore how much of the association between $\mathbf{S}_{\text {het }}$

293 burden and childlessness can be accounted for by the factors described above (where

294 available for the entire cohort), namely: living with a partner, having had sex, having a mental

295 health disorder associated with reduced reproductive success, having a university degree

296 and having an infertility code in health records. Collectively, these factors can account for

297 most of the association of $\mathbf{s}_{\text {het }}$ burden and childlessness in both males $(75 \%)$ and females

$298(65 \%)$, as assessed by the difference in incremental Nagelkerke's $r^{2}$ of deletion $s_{\text {het }}$ burden

299 between models (Methods; Figure 4A; Supplementary Figure 20). By far the biggest

300 contribution comes from living with a partner and having had sex, which together can

301 account for $73 \%$ of the association in males. 
A
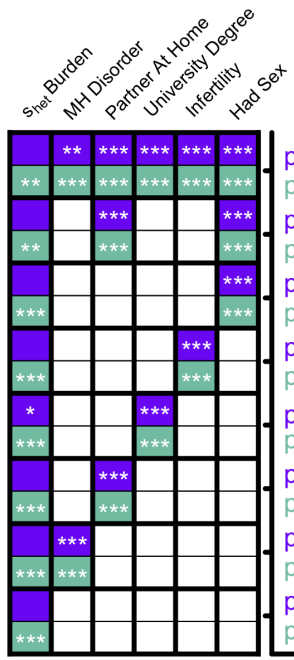

$* p<0.05$

$* * p<0.01$

$* * * p<0.001$
Sex

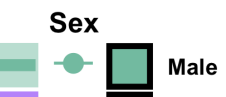

$\begin{array}{ll} & \text { Male } \\ - & \text { Female }\end{array}$ $p=0.13$
$p=9.9 \times 10^{-4}$
$p=0.29$
$p=9 \times 10^{-4}$
$p=0.14$
$p=3.4 \times 10^{-6}$
$p=0.03$
$p=1.5 \times 10^{-1}$
$p=7.1 \times 10^{-3}$

$p=3 \times 10$

$p=8.3 \times 10^{-7}$

$p=0.05$

$p=7.8 \times 10$
$p=0.04$

$=0.04$

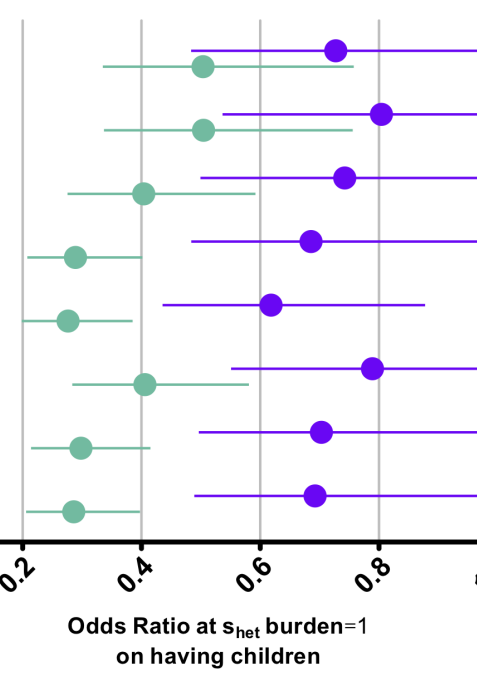

B

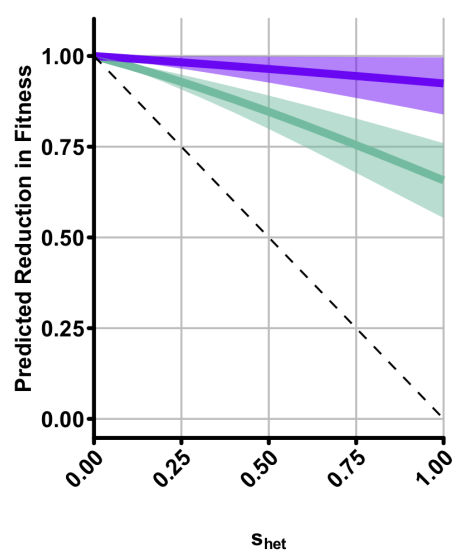

Percent of the Variance Explained by

$S_{\text {het }}$ Compared to the Null Model:

304 Figure 4. The role of individual phenotypes in the relationship between $s_{\text {het }}$ burden and fitness.

305 (A) Odds ratio estimates for the effect of cumulative deleterious variation for a combined

306 meta-analysis (deletions + PTVs) on childlessness (middle), corrected for a combination of whether or

307 not a study participant has a mental health disorder, a partner at home, a university degree, infertility,

308 or ever had sex; traits included in each model are indicated as coloured boxes (males - jade, females

309 - violet) on the $y$-axis. Stars within boxes indicate significance level with childlessness for each

310 covariate independently when correcting for deletion $s_{\text {het }}$ burden. For all possible combinations of

311 these traits, see Supplementary Figure 20. As indicated by coloured boxes, all models include $s_{\text {het }}$

312 burden and were run separately for males (jade) and females (violet). The marginal bar plot to the

313 right gives the proportion of the variance in childlessness explained by $s_{\text {het }}$ burden as calculated for

314 deletions only, scaled to the model which only includes $s_{\text {het }}$ burden (i.e. the model on the bottom of the

315 plot). (B) Predicted reduction in overall fitness as a factor of individual $s_{\text {het }}$ burden. Displayed is the

316 expected reduction in fitness as a factor of increasing $s_{\text {het }}$ burden, independently for each sex. Error is

317 shown as the lighter shaded area surrounding the trend line, and is based on the confidence intervals

318 on the odds ratio as determined by our logistic regression model (Figure 1A; Methods). The dashed

319 line represents the theoretical reduction in fertility as predicted by $s_{\text {het }}{ }^{3}$.

320

321 Overall, we estimate that reduced reproductive success due to $s_{\text {het }}$ burden explains $21 \%$

322 [12-30\%] (Figure 4B; Supplementary Figure 21) of the total reduction in fitness expected due

323 to purifying selection against PTVs as predicted by $\mathbf{s}_{\text {het }}$ (Methods) ${ }^{3}$, with this reduction in

324 fitness being much stronger in males. This suggests that such selection may not be borne

325 equally by males and females. We note that the total reduction in fitness predicted by $\mathbf{s}_{\text {het }}$ will

326 include a substantive contribution from pre-reproductive mortality, which is not quantified

327 here. We also note that current estimates of $\mathrm{s}_{\text {het }}$ are based on data from aggregated

328 research cohorts, and may thus be biased upwards. This is because individuals with high $\mathbf{s}_{\text {het }}$

329 burden are likely to be under-represented within research cohorts (since participation in

330 research has been shown to be biased with respect to gender, socioeconomic status and

331 genetic variation ${ }^{37}$ ), so PTVs within genes under strong selective constraint may well be

332 segregating at higher frequencies in the general population than in research cohorts. This

333 bias could result in the true value of $\mathbf{s}_{\text {het }}$ being lower than currently estimated, and, 
bioRxiv preprint doi: https://doi.org/10.1101/2020.05.26.116111; this version posted December 8,2020 . The copyright holder for this preprint (which was not certified by peer review) is the author/funder, who has granted bioRxiv a license to display the preprint in perpetuity. It is made available under aCC-BY 4.0 International license.

334 consequently, the contribution of reduced reproductive success to the overall reduction in fitness due to purifying selection being greater than estimated here.

337 These estimates of reproductive success and selection coefficients are inevitably reflective

338 of a population at a particular point in time. The proportionate contribution of reduced

339 reproductive success to the overall reduction in fitness associated with genic purifying

340 selection is likely to change over time. Medical advances over recent decades have altered

341 the landscape of infertility and pre-reproductive mortality substantially. Moreover, overall

342 childlessness is highly dynamic over time. Demographic data demonstrate that

343 population-wide childlessness can double in just two decades, a nationwide trend that is

344 readily apparent in UK Biobank (Supplementary Figure 1). We cannot discount that

345 sex-biased sociodemographic factors, in addition to sexual selection, could also be contributing to dampening the apparent association between $s_{\text {het }}$ burden and childlessness in

347 women. Higher educational attainment has been shown to be one of the factors most

348 strongly positively associated with childlessness in a female-biased manner ${ }^{26}$. Indeed, when

349 we correct the association between $s_{\text {het }}$ burden and childlessness for having a university

350 degree, we see a more significant effect of $s_{\text {het }}$ burden on childlessness in females (OR=0.62

351 [95\% Cl 0.44-0.88], $p=7.1 \times 10^{-3}$; Figure 4A; Supplementary Figure 20); but the effect on male

352 childlessness remains considerably stronger than in females.

354 In summary, we find that reduced reproductive success, especially in males, makes a

355 substantial contribution to purifying selection acting on human genes, and that this is likely

356 mediated primarily by mate choice on cognitive and behavioural traits. Mate preferences are

357 multi-dimensional, and vary across cultures and time ${ }^{23}$. It is likely that male-biased reduced

358 reproductive success associated with increased $\mathrm{s}_{\text {het }}$ burden involves multiple cognitive and

359 behavioural traits. The negative impact of $s_{\text {het }}$ burden on fluid intelligence, household income

360 and educational attainment, together with the previously documented female-biased

361 preference for mates with good financial prospects ${ }^{21}$ suggest that sex-biased mate

362 preferences contribute in part to the sex-bias in reproductive success with increased $\mathrm{s}_{\text {het }}$

363 burden. However, as we are not able to assess the effect of $\mathbf{s}_{\text {het }}$ burden on all characteristics

364 that are valued in a mate, especially those that are ranked most highly by both sexes (e.g.

365 emotional stability and maturity ${ }^{21}$, we cannot exclude the possibility that sex biases in the

366 impact of $s_{\text {het }}$ burden on these traits also contribute to the sex bias in reproductive success.

367 Nonetheless, this study represents an important validation of the relevance of Darwin's

368 theory of sexual selection ${ }^{5}$ to contemporary human populations.

370 We note that while this study demonstrates that rare heterozygous genetic variation has a

371 bigger impact on reproductive success in males than females, heritability analyses have

372 suggested a greater contribution of common genetic variation to variance in reproductive

373 success in females than males ${ }^{38}$. These two observations are potentially complementary: the

374 larger contribution of heterozygous rare genetic variation to male reproductive success could

375 be lowering the proportionate contribution of common genetic variation. Previous work

376 demonstrated that the proportion of the genome that is homozygous is also associated with

377 decreased reproductive success through increased childlessness, although without an

378 apparent sex-bias, and proposed that this association is largely driven by rare homozygous

379 variation, which we did not assess here ${ }^{39}$. Involuntary childlessness can have serious 
bioRxiv preprint doi: https://doi.org/10.1101/2020.05.26.116111; this version posted December 8,2020 . The copyright holder for this preprint (which was not certified by peer review) is the author/funder, who has granted bioRxiv a license to display the preprint in perpetuity. It is made available under aCC-BY 4.0 International license.

380 consequences for mental health, and further studies of the genetic contributions to

381 involuntary childlessness are warranted.

382

383 These findings may help to explain, at least in part, why only a minority of genes under the

384 highest selective constraint have been associated with single gene disorders that increase

385 pre-reproductive mortality or cause infertility. While there are clearly many more single gene

386 disorders to be discovered among these genes ${ }^{40,41}$, we anticipate that these highly

387 constrained genes will not be neatly divided into those that cause single gene disorders and

388 those that impact on reproductive success without causing a clinical condition. Rather, we

389 predict that damaging variants in many of these genes will perturb neurodevelopment

390 resulting in a broad spectrum of cognitive and behavioural outcomes, which will increase an

391 individual's risk of childlessness, but only in some cases result in a clinically-ascertainable

392 condition.

394 When investigating sex-biased patterns of genetic associations for cognitive and behavioural

395 traits, the potential contribution of reproductive success and mate choice ought to be

396 considered. For example, it has been posited that the preferential transmission from mothers

397 of inherited alleles increasing risk of neurodevelopmental disorders potentially relates to the

398 greater 'resilience' of females to such alleles ${ }^{42}$. However, our findings that the impact on

399 cognition of the damaging genetic variation studied here is similar between the sexes

400 suggests that, other than for autism spectrum disorder ${ }^{43}$, mate choice may be a more

401 plausible explanation for such observations, as seen for the 22q11.2 deletion ${ }^{44}$.

402

403 These analyses have several limitations. First, we do not have longitudinal relationship data

404 for UK Biobank participants that might shed more light on the impact of $\mathbf{s}_{\text {het }}$ burden on the

405 ability to attract a partner during peak reproductive years. Second, we have not been able to

406 explore the impact of $s_{\text {het }}$ burden on the full range of cognitive and behavioural traits that

407 relate to mate preferences and influence reproductive success. We anticipate that teasing

408 out the relative contributions of correlated cognitive and behavioural traits will be

409 challenging. Third, UK Biobank participants are biased towards higher health, educational

410 attainment and socioeconomic status ${ }^{37}$, and as such the estimates of the negative effect of

$411 \mathbf{S}_{\text {het }}$ burden on reproductive fitness possibly underestimate the true effects in the general

412 population. Finally, we cannot completely account for as-yet-undiscovered male infertility

413 genes in these analyses; nonetheless, these results - in particular those based on clinically-

414 and self-reported health outcomes (Figure 2; Supplementary Figure 11) - suggest a minor contribution of male infertility to the relationship between $s_{\text {het }}$ burden and childlessness.

417 Our study focused on individuals of European ancestry and analogous studies across

418 different populations and cultures are needed. Males have considerably greater variance in

419 reproductive success than females across cultures ${ }^{45}$, including higher levels of childlessness

420 than females ${ }^{26}$, highlighting the potential for sexual selection acting on male reproductive

421 success to act across populations. We also note that many of the fundamental trends

422 relating to mate preferences and male childlessness have been shown to be cross-cultural in

423 nature ${ }^{21,25,45}$. We anticipate future studies that integrate genome-wide sequencing data on

424 large population samples from a range of ancestries to more fully characterise the impact of

425 sexual selection on our species. 


\section{Methods}

\section{Sample Selection and Phenotype Collation}

428 To collate phenotypes for all individuals in UK Biobank, we downloaded bulk phenotype files

429 from the UK Biobank data showcase (https://www.ukbiobank.ac.uk/data-showcase/; data

430 acquired 22 Jan 2020). Due to ascertainment biases with post-recruitment data

431 (Supplementary Figure 18), we only retained data which were ascertained at time of

432 recruitment as opposed to those ascertained via followup (i.e. instance 0 in the UK Biobank

433 data showcase). Please see Supplementary Table 1 for detailed descriptions of all

434 phenotypes assessed in this manuscript, including how they were processed, if applicable.

435 Individuals with missing data for a relevant phenotype were excluded from analysis when

436 testing that phenotype.

437

438 Following phenotype collation, we next selected for final analysis individuals of broadly

439 European ancestry as determined by Bycroft et al. ${ }^{46}$, which left a total of 409,617 individuals.

440 To identify and remove related individuals, we first downloaded the relatedness file from the

441 UK Biobank data showcase using the ukbbgene tool, which contains 107,124 relatedness

442 pairs among UK Biobank participants ${ }^{46}$. Next, we sorted individuals by the total number of

443 related pairs within this file, and removed the individual with the most related pairs and

444 recalculated the total number of relationships for all other individuals. We repeated this

445 process until no related pairs remained, which left a total of 342,717 individuals for

446 downstream analysis.

\section{Calling, Quality Control, and Annotation of Copy Number 448 Variants from SNP Microarrays}

449 To ascertain copy number variants from 488,377 UK Biobank participants with available 450 genetic data ${ }^{46}$, we utilised the PennCNV CNV-ascertainment pipeline ${ }^{47}$. Raw CEL files were 451 downloaded in 107 independent batches, of which 95 batches were genotyped with the 452 standard UK Biobank array platform and 12 batches were genotyped with the UKBiLEVE 453 array platform. Each batch was then processed independently through the following calling 454 pipeline: first, raw CEL files were genotyped with Affymetrix power tools

455 (http://media.affymetrix.com/support/developer/powertools/changelog/index.html) 'genotype'

456 with default settings. Next, using the 'generate_affy-geno_cluster.pl' and

457 'normalize_affy_geno_cluster.pl' scripts provided as part of PennCNV, genotyped samples

458 within each batch were clustered and normalised, respectively. Normalised clustering output

459 was then split into one file per individual and provided as input to 'detect_cnv.pl' to generate

460 an initial call set of CNVs. Finally, initial CNVs were then passed to the 'clean_cnv.pl' script

461 with "-fraction" set to 0.25 in order to merge nearby CNV calls in each individual. Following

$462 \mathrm{CNV}$ calling, we excluded all individuals with $\geq 20 \mathrm{CNVs}$ and absolute waviness factor $>0.3$,

463 and all variants on either the $X$ or $Y$ chromosome, which left 485,593 individuals and

$4643,101,974$ raw, redundant CNVs. 
bioRxiv preprint doi: https://doi.org/10.1101/2020.05.26.116111; this version posted December 8,2020 . The copyright holder for this preprint (which was not certified by peer review) is the author/funder, who has granted bioRxiv a license to display the preprint in perpetuity. It is made available under aCC-BY 4.0 International license.

466 To perform quality control of ascertained CNVs, we developed a novel approach which uses

467 individuals for which CNVs have been ascertained with both array and exome-based

468 approaches. In short, we started with the basic logistic regression concept outlined in Mace

469 et al. ${ }^{48}$ but instead used the intersect of array- and WES-ascertained CNVs as the

470 dependent variable in a random forest model ${ }^{49}$, with various per-individual and per-CNV

471 metrics as predictors. To train this model, we utilised an additional set of 46,856 individuals

472 collected as part of the INTERVAL study ${ }^{50}$ genotyped on the same array as participants in

473 UK Biobank, of which 4,465 also had matched WES data. For INTERVAL individuals, we

474 performed array-based CNV calling identically to the method as described above and

475 ascertained exome-based CNVs using three different algorithms with default settings:

$476 \mathrm{XHMM}^{51}, \mathrm{CANOES}^{52}$, and CLAMMS ${ }^{53}$. For each INTERVAL participant for which we had

477 both array and exome-based CNVs, we then determined a "WES overlap score" as a

478 product of the overlap of each array-based CNV with the three WES-based callers, corrected

479 for whether or not any overlap was possible due to probe/exon bias. Scoring results in a

480 roughly continuous metric for each array-ascertained CNV of between zero and three, where

481 zero represents a lack of overlap with any WES CNV call and three represents a perfect

482 overlap with all three algorithms. For predictor covariates, we used several metrics already

483 shown to be of high quality for CNV quality control ${ }^{48,54}$, per-CNV metrics based on these (e.g.

484 mean log $\mathrm{R}$ ratio for each probe within a CNV rather than for all probes across an entire

485 individual), and a novel metric which uses specific probes on the array known to be biased

486 for CNV calls on bad arrays (Supplementary Table 3; see code availability). To determine

487 estimated sensitivity/specificity of our model we performed 10 -fold cross-validation, where all

488 array CNVs which overlapped at least two exons were split into equal test and training sets

489 and provided, separately for deletions and duplications, as input into the randomForest

490 implementation in $\mathrm{R}$ as a linear predictor with nTrees set to 500 . To generate a call set of

491 final quality controlled CNVs for downstream analyses, we then trained a final random forest

492 using all INTERVAL individuals with matched array and WES data and generated predicted

493 WES overlap scores for all 3,101,974 raw UK Biobank CNVs identified with PennCNV as

494 described above. CNVs were then filtered based on a predicted sensitivity of $95 \%$ based on

495 cross-validation, leaving a remaining 1,612,931 CNVs (1,043,717 deletions, 569,114

496 duplications).

497

498 CNVs passing quality control were then provided as input to a custom java pipeline which

499 merged all CNVs, regardless of whether they were deletions or duplications, based on $75 \%$

500 reciprocal overlap to generate a set of 173,871 nonredundant loci. Following filtering to

501342,717 unrelated individuals of broadly European ancestry for which CNV data was

502 available, each locus was quantified for allele frequency. Loci were then assessed for

503 overlap with a set of known pathogenic CNVs identically to Crawford, et al ${ }^{54}$ and annotated

504 using Variant Effect Predictor (VEP) v97 ${ }^{55}$. Only loci with an annotation of

505 'transcript_ablation' or 'feature_trunctation' and 'coding_sequence_variant' for deletions, and

506 'transcript_amplification' or 'feature_elongation' and 'coding_sequence_variant' for

507 duplications were considered to be affecting a target gene. A total of 1,118,859 redundant

$508 \mathrm{CNVs}$ remained for downstream analysis following all filtering and annotation $(721,536$

509 deletions, 397,323 duplications; Supplementary Figure 2). 


\section{Processing SNV/InDel Data from WES}

511 To collate protein truncating, missense, and synonymous variants for all 49,960 individuals

512 whole exome sequenced by UK Biobank ${ }^{11}$, we downloaded the GRCh38-aligned

513 population-level variant files from the UK Biobank (UK Biobank field 23160) and converted

514 them to variant call format. All autosomal variants were then annotated with VEP $\mathrm{v} 97^{55}$,

515 CADDv1.5 ${ }^{56}$, allele frequency from gnomAD ${ }^{57}, \mathrm{PEXT}^{58}$ and, where relevant, MPC ${ }^{59}$ and

516 LOFTEE $^{57}$. PEXT and MPC scores were converted from build37 to build38 using the

517 CrossMap tool ${ }^{60}$. Variants were assigned to a gene based on the primary ENSEMBL

518 transcript with the most severe consequence. Variants were considered to be PTVs if they

519 were annotated by VEP as having a splice acceptor/donor, stop gained, or frameshift

520 consequence. We then retained only variants with a gnomAD or UK Biobank-specific allele

521 frequency $\leq 1 \times 10^{-3}$ and with a PEXT mean cerebellum score $>0.1$. Missense variants were

522 only retained if they had MPC $>2$ and CADD $>25$. PTVs were only retained if they were

523 annotated by LOFTEE as high confidence, had CADD $>25$, and were not located in the last

524 exon or intron of the canonical transcript as annotated by ENSEMBL ${ }^{61}$. This filtering

525 approach left a total of 2,658,431 redundant autosomal SNVs and InDels across all 34,812

526 unrelated individuals of broadly European ancestry included in this study (Supplementary

527 Figure 3).

528

529 It has recently been reported that the UK Biobank exome sequencing data is missing variant

530 calls in regions where all reads were assigned $M A P Q=0$ (for more details, see Jia et al. ${ }^{62}$ ).

531 While this issue affects 703 genes with an $s_{\text {het }}$ value assessed in this study, genes with the

532 highest constraint scores (i.e. $\left.s_{\text {het }} \geq 0.15\right)$ are less likely to be affected by this problem ( $3.3 \%$

533 of genes with $s_{\text {het }} \geq 0.15,4.5 \%$ of genes with $s_{\text {het }}<0.15$; Fisher's $p=0.02$ ). Secondly, this

534 issue is consistent across all individuals with WES within the UK Biobank and thus results in

535 a simple loss of power equivalent to having insufficient coverage to call variants across $\sim 4 \%$

536 of the exome. Finally, as CNV calling was performed using genotyping arrays, and thus

537 unaffected by issues with sequence alignment, our findings are independently robust.

538 Information on exome capture baits and genes affected by alignment issues for producing

539 this statement were acquired from the UK Biobank data showcase

540 (http://biobank.ctsu.ox.ac.uk/crystal/refer.cgi?id=1911).

\section{Calculating $\mathbf{s}_{\text {het }}$ Burden for UK Biobank Participants}

542 To calculate an individual's $\mathbf{s}_{\text {het }}$ burden, assuming that fitness is multiplicative and that there

543 is no epistasis between genes which are lost, we utilised the following formula:

$$
s_{h e t[i, v]}=1-\prod_{g}\left(1-s_{h e t[i, v, g]}\right)
$$

545 where $s_{\text {het }[i, v]}$ indicates individual $i$ 's $\mathrm{s}_{\text {het }}$ burden for variant class $v$ and $s_{\text {het }[i, v, g]}$ indicates the $546 \mathrm{~s}_{\text {het }}$ score for gene $g$ with a qualifying annotation for variant class $v$ in individual $i$. Possible 547 values for $v$ are PTV, missense, synonymous, deletion, or duplication. As indicated by the 548 formula above, $s_{\text {het }}$ values were calculated independently for each variant type. Per-gene $s_{\text {het }}$ 549 values were obtained from Weghorn et al. ${ }^{3}$, under their demographic model which includes 
550 drift and scores for 16,189 protein coding genes which we were able to definitively map to an

551

583 All models were run separately for males and females. For binary phenotypes, 'family' was

ENSEMBL gene ID. To ensure that our primary result of the effect of $s_{\text {het }}$ burden on childlessness is unaffected by the version of $s_{\text {het }}$ we use to calculate our burden scores, we also utilised an earlier derivation of $\mathrm{s}_{\text {het }}$ from Cassa et al. ${ }^{4}$ which does not take into account a demographic model. This did not significantly change our primary result (Supplementary Figure 22).

To explore if genes known to be associated with male infertility were responsible for our observed effect on male reproductive success, we also generated individual $s_{\text {het }}$ scores for each variant class excluding a set of 150 autosomal genes known to be associated with male infertility (Supplementary Table 5 from Oud et al. ${ }^{14}$ ). Genes with an annotation of limited, moderate, strong, or definitive evidence were excluded from calculated $\mathrm{s}_{\text {het }}$ scores $^{15}$. Similarly, and to test if a greater number of 742 genes associated with male infertility in mice were responsible for our observed effect on male reproductive success, we queried all genes from Mouse Genome Informatics ${ }^{15}$ with a phenotype code of MP:0001925. Gene IDs were then translated to their human homologues and $\mathrm{s}_{\text {het }}$ burden scores excluding these genes were then generated and provided as input to logistic regression as described above.

To test if our observed relationship was robust when excluding genes with a known disease annotation, we also generated individual $s_{\text {het }}$ scores where we removed 4,414

disease-associated genes. We considered a gene to be disease-associated based on being a confirmed or probable developmental disorder gene in the Developmental Disorders Genotype-Phenotype Database (DDG2P; https://decipher.sanger.ac.uk/info/ddg2p), in the Online Mendelian Inheritance in Man (OMIM; https://omim.org/) Morbid Map after excluding 'non disease' and 'susceptibility to multifactorial disorder' entries, or in $\mathrm{ClinVar}^{63}$ with a pathogenic/likely pathogenic variant linked to a phenotype.

\section{Logistic and Linear Modelling of Phenotypes}

To test the association of each $\mathrm{s}_{\text {het }}$ burden (i.e. $s_{\text {het }[i, v]}$ ) per variant class with a given phenotype (e.g. those in Supplementary Table 1), we used a general linear model via the ' $g l m$ ' function in R of the form:

set to 'binomial' and for continuous phenotypes 'family' was set to 'gaussian'. To combine the effect sizes or log odds ratios for CNVs and PTVs (e.g. for Figure 1A), we used the 'metagen' function from the 'meta' package ${ }^{64}$ in $\mathrm{R}$ to perform a fixed-effects meta analysis. For logistic regression, we set parameters 'method.tau' to 'SJ' and 'sm' to 'OR'. For linear regression, we set the parameter 'sm' to "SMD". To avoid including an individual twice in our meta analysis, for samples with both CNV and PTV data available, we prioritised PTV-derived $s_{\text {het }}$ scores. 
592 When using raw variant counts as in Supplementary Figure 7, the $s_{\text {het }}$ term in the above

593 formula was changed to the total number of qualifying genes affected per individual, where

594 qualifying genes were either those with $\mathrm{pLI} \geq 0.9^{57}$ or those with $\mathrm{s}_{\text {het }} \geq 0.15^{3}$. Individuals with

$595>3$ genes lost for deletions ( $\left.p L I \geq 0.9 n=43 ; s_{\text {het }} \geq 0.15 n=16\right)$ and PTVs $(p L I \geq 0.9 n=2$;

$596 s_{\text {het }} \geq 0.15 n=1$ ) were removed prior to regression analyses. To provide a negative control

597 for our association tests, we also performed associations for several neutral phenotypes we

598 hypothesised to not be under negative selection: fresh fruit intake, handedness, and blonde

599 hair colour (Supplementary Table 1). None of these associations were significant after

600 correcting for multiple testing (Supplementary Figure 23).

601

602 To test the effect of individual phenotypes on likelihood of having children (Supplementary

603 Figure 14), we used a logistic model (with the 'family' parameter of the 'glm' function set to

604 'binomial' in R) of the form:

605

606

$$
\text { has.children } \sim \text { phenotype }+ \text { age }+ \text { age } e^{2}+P C 1 . . P C 30
$$

607

608 As with estimating the contribution of $s_{\text {het }}$ burden to phenotypes, all analyses were run

609 separately for both males and females. For all models involving household income, we

610 additionally included partner at home status as a covariate, as household income was

611 recorded per household, not per recruited individual.

612

613 Pre-computed ancestry principal components for each UK Biobank participant were taken

614 from Bycroft et al. ${ }^{46}$. To alleviate concerns about a potentially arbitrary selection of the

615 number of ancestry principal components used in our models, we repeated our primary

616 analysis of the association between having children and $s_{\text {het }}$ burden in males with between

61710 and 30 ancestry principal components and did not observe any significant differences in

618 our result (Supplementary Figure 24).

619

620 All odds ratios, effect sizes, standard errors, $p$ values, and total individuals per association

621 test reported in this manuscript can be found in Supplementary Table 4.

\section{Collation and Testing of Participant Medical Data}

623 To assess if a broad range of medical conditions play a role in mediating the effect of high

$624 \mathrm{~S}_{\text {het }}$ burden on childlessness, we queried two relevant datasets provided by the UK Biobank ${ }^{10}$ :

625 hospital episode statistics (HES) and combined health outcomes data (CHOD;

626 Supplementary Table 1). Briefly, for each UK Biobank participant, HES data incorporates

627 electronic inpatient data provided directly from NHS hospitals and CHOD aggregates HES,

628 general practitioner records, self-reported conditions, and death records. All data sources

629 are coded according to the International Classification of Disorders v10 (ICD-10). For the

630 purposes of this work, we ignored all cancer codings from HES and CHOD data (ICD

631 chapter II and codes $000-008$ of chapter XV), and instead used independent cancer registry

632 data; the UK Biobank acquires information on cancer diagnoses from the UK cancer registry

633 which aggregates a wide range of data sources including general practitioners, nursing

634 homes, and hospitals and is considered the more accurate data source for UK Biobank 
bioRxiv preprint doi: https://doi.org/10.1101/2020.05.26.116111; this version posted December 8, 2020. The copyright holder for this preprint (which was not certified by peer review) is the author/funder, who has granted bioRxiv a license to display the preprint in perpetuity. It is made available under aCC-BY 4.0 International license.

662 Where icd.code represents a binarised presence/absence of one of 19,154 different ICD-10

\section{Evaluation of Gene Expression in Testis}

675 To determine the expression in testis of all genes assessed in this study, we downloaded 676 processed median transcripts per million values for all genes provided by $v 7$ of the GTEx 677 study

participant cancer diagnoses. Cancer codes were retained only when testing HES data (Figure 2, Supplementary Figures 11-13).

We utilised both HES and CHOD sources to examine a broad set of medical conditions in UK Biobank participants. Complete HES are available for all UK Biobank participants but are probably depleted of conditions that are unlikely to be seen in a hospital setting (e.g. male infertility). CHOD are also likely to be more sensitive to a wide variety of conditions as they incorporate aforementioned HES data with both general practitioner records and self-reported outcomes. Importantly, while general practitioner records are only available for $46 \%(n=230,090)$ of UK Biobank participants, when we tested for an association between $\mathrm{s}_{\text {het }}$ burden and whether a participant had general practitioner records or not, we did not observe a significant association for either males ( $\mathrm{OR}=1.02$ [95\% $\mathrm{Cl} 0.75-1.37], \mathrm{p}=0.92)$ or females (OR=1.26 [95\% Cl 0.94-1.68], $p=0.12$; Supplementary Figure 18). This indicated that we were unlikely to see biases due to including CHOD from individuals who were missing general practitioner records. As such, we prioritised the use of CHOD in most analyses presented in the text, figures, and supplementary information of this manuscript complete results for all codes in both HES and CHOD data are available as Supplementary Table 2. Exceptions include when testing codings from chapters XVII to XXII which are beyond the diagnostic scope of CHOD, and cancer codes better ascertained from the UK Biobank cancer registry as noted above (Figure 2; Supplementary Figures 12, 13).

To determine the role of 19,154 diseases, disorders, and special codes collated from HES and $\mathrm{CHOD}$ in the relationship between $\mathrm{s}_{\text {het }}$ burden and childlessness (Figure 2), we used a modified version of our primary logistic model of the form:

$$
\text { has.children } \sim s_{\text {het }[i, v]}+i c d . c o d e_{c}+\text { age }+ \text { age } e^{2}+P C 1 . . P C 30
$$
diseases, disease groups, and chapters, $c$. Tests were only performed when a given code was represented by at least 2 individuals in both genetic (i.e. CNVs) and WES (i.e. PTVs) data. When considering individuals who have a particular code, $c$, we utilised the hierarchical information present within the ICD-10 coding system. For example, when we tested if inclusion of a term for individuals with non-insulin-dependent diabetes mellitus (ICD-10 code E11) has an effect on childlessness, we also considered individuals with any sub-code (i.e. E11.0-E11.9). This same principle was also used for disease groupings when testing the more general diabetes mellitus group (ICD-10 block E10-E14) we included all individuals with any code between E10 and E14, including disease subtypes (e.g. E11.0). For each model, we retained both an odds ratio for the effect of individual $\mathrm{s}_{\text {het }}$ burden and presence of a given code on childlessness (Supplementary Table 2). 
bioRxiv preprint doi: https://doi.org/10.1101/2020.05.26.116111; this version posted December 8,2020 . The copyright holder for this preprint (which was not certified by peer review) is the author/funder, who has granted bioRxiv a license to display the preprint in perpetuity. It is made available under aCC-BY 4.0 International license.

678 (https://storage.googleapis.com/gtex_analysis_v8/rna_seq_data/GTEx_Analysis_2017-06-0

679 5_v8_RNASeQCv1.1.9_gene_median_tpm.gct.gz). Only genes for which an $\mathrm{s}_{\text {het }}$ score was

680 available $^{3}$ were retained from this file. We then determined if each gene was affected by

681 either a private deletion or PTV in a UK Biobank individual. We then plotted

682 In (testis expression) in two ways: (i) as a factor of being a male infertility gene or not or (ii)

683 having or not having a qualifying variant (Supplementary Figure 10). To determine

684 significance we used a one-sided Wilcoxon test, with the alternate hypothesis that

685 expression in testis of male infertility genes or genes with private variants is greater than the

686 alternative set.

\section{Modelling the Contribution of Phenotypes to Observed \\ 688 Reduction in Fitness}

689 Variant $\boldsymbol{s}_{\text {het }}$ Burden

690 To estimate the contribution of $s_{\text {het }}$ to overall fitness (Figure 4B), we extracted log odds ratio

691 estimates for the effect of $\mathrm{S}_{\text {het }}$ on having children from our logistic model and estimated the

692 proportion of childless individuals at various $s_{\text {het }}$ scores ( 0 to 1 at 0.1 intervals;

693 Supplementary Figure 21). To calculate the error in our estimates (i.e. the shaded areas in

694 Figure 4B), we used the $95 \%$ confidence intervals for the $s_{\text {het }}$ burden log odds ratio from our

695 original logistic regression. Please see Supplementary Note 1 for a more detailed description

696 of how the contribution of $\mathbf{s}_{\text {het }}$ to overall fitness was calculated.

697

\section{General Cognition}

699 When possible, we used independent estimates from population level or external data to

700 alleviate biases in UK Biobank phenotype ascertainment (Supplementary Figure 18) ${ }^{35}$. As

701 such, data on cognitive ability and fertility are collected from Swedish population-level

702 government administrative registers that have been linked to Swedish conscription

703 registers $^{65}$. To assess assignment into different branches of a universal conscription for

704 Swedish men, the Swedish government included an extensive cognitive ability test which all

705 men in Sweden had to take part in. Information on childbearing is based on birth records,

706 and linkage to both men and women is nearly universal, partly due to universal government

707 identity numbers, combined with serious paternity investigations in case of missing

708 information of the biological father. This information was used to calculate reproductive

709 fertility histories in 2012 for all men included in this study. We include data on all Swedish

710 born men who participated in the military conscription test at age 18-20 who were born

711 1965-1967. The conscription registers are described in more detail elsewhere ${ }^{32,66}$.

713 For the current study, we did not rely on the official cognitive ability scores assigned for each

714 man following their cognitive ability test as in Kolk and Barclay ${ }^{67}$, but instead made manual

715 calculations to create a more finely grained measure from raw test scores based on a battery

716 of cognitive ability tests that are available for 3 years in our conscription registers. The

717 Swedish military created an official IQ-measure based on a 9-score stanine scale that has

718 been used in a large number of scientific studies ${ }^{67,68}$. In the current study we developed a

719 more detailed score using information on the actual test scores of men participating in the

720 test. The conscription test consisted of four large subtests measuring different dimensions of

721 IQ with logical, spatial, verbal, and technical subtest ${ }^{66,69,70}$. To get a more finely tuned IQ 
bioRxiv preprint doi: https://doi.org/10.1101/2020.05.26.116111; this version posted December 8,2020 . The copyright holder for this preprint (which was not certified by peer review) is the author/funder, who has granted bioRxiv a license to display the preprint in perpetuity. It is made available under aCC-BY 4.0 International license.

731

732 This score allows us to calculate cognitive ability by single digit IQ scores (Supplementary

733 Table 5); however, as we had to rely on only observations with complete test scores for all

734 test batteries, our data has a higher share of excluded men than the official cognitive ability

735 scores (used by Kolk and Barclay ${ }^{32}$ and others). In addition to the $\sim 5 \%$ of men that did not

736 take the test (e.g. they were ineligible for military service due to handicap such as visual

737 impairments, that they were abroad, or were conscripted at an atypical age), we additionally

738 excluded a number of men for which scores of all test batteries were not available. Our

739 manually computed fine-grained measure was later standardised against the official

740 cognitive ability test score to maintain comparability and to assure our slightly smaller

741 population is still representative of the complete cohort. Compared to most other measures

742 of cognitive ability in the scientific literature, we argue that our population is unusually

743 representative as little (indirect) pre-selection due to cognitive ability took place.

744

745 We first estimated the effect of overall $s_{\text {het }}$ burden on fluid intelligence (Figure 3F) and,

746 because fluid intelligence is normalised and IQ is normally distributed, converted this effect

747 to a predicted change of IQ. To then estimate childlessness and fertility for low IQ values not

748 actually observed in the general population, we fit actual observations to a sigmoidal model

749 using the function 'nls' in R (Supplementary Figure 17; Supplementary Table 5). As our

750 empirical distribution did not conform to a standard test distribution, we then simulated

751100,000 individuals, with IQ values for each individual randomly selected from our original

752 Swedish IQ distribution with the mean shifted by the expected reduction in IQ as explained

753 by our $\mathbf{s}_{\text {het }}$ model. We then assigned each simulated individual an expected number of

754 children and predicted probability of childlessness based on their simulated IQ value as

755 given in Supplementary Table 5. Number of children across all 100,000 individuals was then

756 averaged to generate an expected mean fertility for a given $\mathbf{s}_{\text {het }}$ score (Supplementary Figure

757 16). This value was then compared to the mean number of children for the unburdened

758 population via the following formula:

760 To then calculate the proportion of reduced reproductive success explained by IQ (and by 761 extrapolation, other traits) we then divide this fertility ratio by the overall reduction in fitness

762 given by $\mathbf{s}_{\text {het }}$ as described above. Please see Supplementary Note 2 for a more detailed 763 example of how we performed this calculation.

764

765 Mental Health Disorders

766 As with general cognition, we used estimates from external studies to alleviate biases in UK

767 Biobank phenotype ascertainment (Supplementary Figure 18$)^{35}$. In this case, as we were 
768 unable to accurately estimate the increased risk of developing individual mental health

807 Relatively complete mental health disorder data are available for all individuals via the 808 complete health outcomes data; therefore, we also included a covariate for having a mental disorders as a factor of individual $s_{\text {het }}$ burden, we instead utilised odds ratios from Ganna et al. ${ }^{9}$. Only odds ratios for schizophrenia, autism spectrum disorder, and bipolar disorder were retained. As Ganna et al. ${ }^{9}$ estimated the risk based on total count of high pLI $(\geq 0.9)^{57}$ genes with PTVs per individual instead of with $s_{\text {het }}$, we assumed that an individual carrying one such variant had an $s_{\text {het }}$ burden of 0.162 , or the mean $s_{\text {het }}$ value of all high pLI $(\geq 0.9)$ genes. We then converted this into a proportion of individuals with a given mental health disorder, $t$, at $\mathrm{s}_{\text {het }}$ burden, $x$, by scaling the odds ratio with the following formula:

$$
\log \left(O R_{s_{\text {het }}[x, t]}\right)=\frac{\log \left(O R_{\text {ganna }}\right) * s_{\text {het }}[x]}{0.162}
$$

To establish a baseline expectation for the prevalence of each mental health disorder at $\mathrm{s}_{\text {het }} 0$ we utilised population-level data from Power et al. ${ }^{19}$ and extrapolated for each trait at increasing $s_{\text {het }}$ values (Supplementary Figure 25). To generate an expected mean number of children for simulated individuals with mental health disorders, we used fertility statistics generated by Power et al. ${ }^{19}$. As Power et al. ${ }^{19}$ did not provide childlessness data, we were unable to generate expected childlessness as we did for other traits. Overall predicted reduced fitness attributable to mental health and all values used for performing the above analyses are provided in Supplementary Table 6.

\section{Having a Partner at Home, Having had Sex, Educational Attainment, and Infertility}

To determine the contribution of having a partner at home, ever having had sex, educational attainment, and a medical diagnosis of infertility to the relationship between $s_{\text {het }}$ burden and childlessness, we utilised a multiple regression model incorporating various combinations of these traits (Figure 4A; Supplementary Figure 20). First, we calculated the variance in childlessness explained by a null model consisting of age, age ${ }^{2}$, and the first 30 ancestry PCs using Nagelkerke's pseudo- $r^{2}$ as calculated using the "nagelkerke" function from the R package "rcompanion" (https://rcompanion.org/handbook/). Next, to determine the proportion of variance explained in childlessness by $s_{\text {het }}$ alone, we calculated incremental pseudo-r ${ }^{2}$ between this null model and a model additionally incorporating a term for deletion $\mathbf{s}_{\text {het }}$ burden. We then repeated this analysis, except now including an additional covariate (e.g. having a partner at home) to determine the reduction in variance explained by deletion $\mathrm{s}_{\text {het }}$ when correcting for the additional covariate. This reduction in variance was then converted to a percent change via the following formula:

$$
\% \text { reduction in variance explained by deletion } s_{\text {het }}=\frac{s_{\text {het }} \text { incremental } r^{2} \text { with covariate }}{s_{\text {het }} \text { incremental } r^{2} \text { without covariate }}
$$

This basic analysis was then repeated for all possible combinations of having a partner at home, ever having had sex, having a university degree, and having a medical diagnosis of infertility (Figure 4A; Suppelementary Figure 20). Percent reduction in variance explained values plotted in Figure 4A and Supplementary Figure 20 are displayed for $\mathrm{s}_{\text {het }}$ calculated using deletions only. health disorder as a covariate in our multiple regression model (Figure 4A). As income is provided by UK Biobank on a per-household basis (Figure $3 \mathrm{E}$ ) and the number of individuals with fluid intelligence data recorded at recruitment is significantly smaller than for other 2 covariates (Figure 3F), we did not include these as part of our multiple regression model. 


\section{${ }_{813}$ Acknowledgements}

814 We thank Leopold Parts, Molly Przeworski and George Davey-Smith for useful discussions

815 and comments during manuscript preparation. We thank the INTERVAL study for sharing

816 genotyping and exome data that allowed us to refine our CNV filtering methodology. We

817 thank Manon Oud and Joris Veltman for helpful discussions regarding male infertility in mice.

818 We thank the reviewers for constructive comments and criticism. This work has been funded

819 by core Wellcome funding to the Wellcome Sanger Institute (grant WT098051). This work

820 has been conducted using the UK Biobank Resource under application numbers 14421 (to

821 G.K.) and 44165 (to H.C.M.).

\section{${ }_{822}$ Author Contributions}

823 E.J.G, M.D.C.N, and K.E.S. assessed the contribution of rare genetic variation to the

824 phenotypes and vital statistics presented in this manuscript. E.J.G and G.K. performed CNV

825 calling. E.J.G. and M.E.K.N. annotated and assessed SNV and InDel variants from provided

826 WES data. K.B., M.K., E.J.G, and M.E.H. curated and analysed Swedish IQ data. E.J.G.,

827 K.E.S., H.C.M., and M.E.H. designed experiments, oversaw the study and wrote the

828 manuscript.

\section{${ }_{829}$ Data Availability}

$830 \mathrm{CNVs}$, SNVs and InDels included in this study will be returned to the UK Biobank following

831 study publication, as per UK Biobank guidelines.

\section{${ }_{832}$ Code Availability}

833 Code used as part of this project to perform phenotype testing, CNV calling, variant quality

834 control, and generate all main text figures, supplementary figures and supplementary tables

835 is available on github: https://github.com/eugenegardner/UKBBFertility.

\section{${ }_{836}$ References}

837 1. Lek, M. et al. Analysis of protein-coding genetic variation in 60,706 humans. Nature 536, 285-291 (2016).

839 2. Collins, R. L. et al. A structural variation reference for medical and population genetics. Nature 581, 444-451 (2020).

841 3. Weghorn, D. et al. Applicability of the Mutation-Selection Balance Model to Population 
Genetics of Heterozygous Protein-Truncating Variants in Humans. Mol. Biol. Evol. 36, $1701-1710(2019)$

4. Cassa, C. A. et al. Estimating the selective effects of heterozygous protein-truncating variants from human exome data. Nat. Genet. 49, 806-810 (2017).

5. Darwin, C. The descent of man, and selection in relation to sex. By Charles Darwin. (1874) doi:10.5962/bhl.title.16749.

6. Ganna, A. et al. Ultra-rare disruptive and damaging mutations influence educational attainment in the general population. Nat. Neurosci. 19, 1563-1565 (2016).

7. Männik, K. et al. Copy number variations and cognitive phenotypes in unselected populations. JAMA 313, 2044-2054 (2015).

8. Huguet, G. et al. Measuring and Estimating the Effect Sizes of Copy Number Variants on General Intelligence in Community-Based Samples. JAMA Psychiatry 75, 447-457 (2018).

9. Ganna, A. et al. Quantifying the Impact of Rare and Ultra-rare Coding Variation across the Phenotypic Spectrum. Am. J. Hum. Genet. 102, 1204-1211 (2018).

10. Sudlow, C. et al. UK biobank: an open access resource for identifying the causes of a wide range of complex diseases of middle and old age. PLoS Med. 12, e1001779 (2015).

11. Van Hout, C. V. et al. Exome sequencing and characterization of 49,960 individuals in the UK Biobank. Nature 586, 749-756 (2020).

12. Dudel, C. \& Klüsener, S. Estimating men's fertility from vital registration data with missing values. Population Studies vol. 73 439-449 (2019).

864 13. Office of National Statistics. Birth Summary Tables, England and Wales 2019. (2020).

865 14. Oud, M. S. et al. A systematic review and standardized clinical validity assessment of male infertility genes. Hum. Reprod. 34, 932-941 (2019).

867 15. Bult, C. J. et al. Mouse Genome Database (MGD) 2019. Nucleic Acids Res. 47, 
16. Huang, N. et al. A Screen for Genomic Disorders of Infertility Identifies MAST2 Duplications Associated with Nonobstructive Azoospermia in Humans. Biol. Reprod. 93, $61(2015)$.

17. Skjaerven, R., Wilcox, A. J. \& Lie, R. T. A population-based study of survival and childbearing among female subjects with birth defects and the risk of recurrence in their children. N. Engl. J. Med. 340, 1057-1062 (1999).

18. Lie, R. T., Wilcox, A. J. \& Skjaerven, R. Survival and reproduction among males with birth defects and risk of recurrence in their children. JAMA 285, 755-760 (2001).

19. Power, R. A. et al. Fecundity of patients with schizophrenia, autism, bipolar disorder, depression, anorexia nervosa, or substance abuse vs their unaffected siblings. JAMA Psychiatry 70, 22-30 (2013).

20. Allen, M. S. The Role of Personality in Sexual and Reproductive Health. Current Directions in Psychological Science vol. 28 581-586 (2019).

21. Buss, D. M. et al. International Preferences in Selecting Mates: A Study of 37 Cultures. J. Cross. Cult. Psychol. 21, 5-47 (1990).

22. Pawłowski, B. \& Dunbar, R. I. Impact of market value on human mate choice decisions. Proc. Biol. Sci. 266, 281-285 (1999).

23. Buss, D. M. \& Schmitt, D. P. Mate Preferences and Their Behavioral Manifestations. Annu. Rev. Psychol. 70, 77-110 (2019).

24. Fieder, M., Huber, S. \& Bookstein, F. L. Socioeconomic status, marital status and childlessness in men and women: an analysis of census data from six countries. J. Biosoc. Sci. 43, 619-635 (2011).

25. Nettle, D. \& Pollet, T. V. Natural selection on male wealth in humans. Am. Nat. 172, 658-666 (2008).

893 26. Miettinen, A., Rotkirch, A., Szalma, I., Donno, A. \& Tanturri, M.-L. Increasing 
childlessness in Europe: time trends and country differences. (Family and Societies Working Paper 33, 2015).

27. Jalovaara, M. et al. Education, Gender, and Cohort Fertility in the Nordic Countries. Eur. J. Popul. 35, 563-586 (2019).

28. Fieder, M. \& Huber, S. The effects of sex and childlessness on the association between status and reproductive output in modern society. Evolution and Human Behavior vol. 28 392-398 (2007).

29. Barthold, J. A., Myrskylä, M. \& Jones, O. R. Childlessness drives the sex difference in the association between income and reproductive success of modern Europeans. Evolution and Human Behavior vol. 33 628-638 (2012).

30. Bateman, A. J. Intra-sexual selection in Drosophila. Heredity 2, 349-368 (1948).

31. Trivers, R. Parental Investment and Sexual Selection. in Sexual selection and the descent of man (ed. Campbell,

B.) (Aldine, 1972).

907

32. Kolk, M. \& Barclay, K. Cognitive ability and fertility among Swedish men born 1951-1967: evidence from military conscription registers. Proc. Biol. Sci. 286, 20190359 (2019).

33. Kendall, K. M. et al. Cognitive Performance Among Carriers of Pathogenic Copy Number Variants: Analysis of 152,000 UK Biobank Subjects. Biol. Psychiatry 82, $103-110(2017)$.

34. Davis, K. A. S. et al. Mental health in UK Biobank - development, implementation and results from an online questionnaire completed by 157366 participants: a reanalysis. BJPsych Open 6, e18 (2020).

35. Tyrrell, J. et al. Genetic predictors of participation in optional components of UK Biobank. Cold Spring Harbor Laboratory 2020.02.10.941328 (2020) doi:10.1101/2020.02.10.941328.

36. Stefansson, H. et al. CNVs conferring risk of autism or schizophrenia affect cognition in 
controls. Nature vol. 505 361-366 (2014).

921 37. Fry, A. et al. Comparison of Sociodemographic and Health-Related Characteristics of

922 UK Biobank Participants With Those of the General Population. Am. J. Epidemiol. 186,

$923 \quad 1026-1034(2017)$.

924 38. Watanabe, K. et al. A global overview of pleiotropy and genetic architecture in complex

925 traits. Nat. Genet. 51, 1339-1348 (2019).

926 39. Clark, D. W. et al. Associations of autozygosity with a broad range of human

927 phenotypes. Nat. Commun. 10, 4957 (2019).

928 40. Stanley, K. E. et al. Causal Genetic Variants in Stillbirth. N. Engl. J. Med. (2020)

929 doi:10.1056/NEJMoa1908753.

930 41. Kaplanis, J. et al. Evidence for 28 genetic disorders discovered by combining healthcare 931 and research data. Nature 586, 757-762 (2020).

932 42. Girirajan, S. et al. Phenotypic heterogeneity of genomic disorders and rare copy-number 933 variants. N. Engl. J. Med. 367, 1321-1331 (2012).

934 43. De Rubeis, S. et al. Synaptic, transcriptional and chromatin genes disrupted in autism.

$935 \quad$ Nature 515, 209-215 (2014).

936 44. Costain, G., Chow, E. W. C., Silversides, C. K. \& Bassett, A. S. Sex differences in

937 reproductive fitness contribute to preferential maternal transmission of $22 q 11.2$

938 deletions. J. Med. Genet. 48, 819-824 (2011).

939 45. Betzig, L. Means, variances, and ranges in reproductive success: comparative

940 evidence. Evolution and Human Behavior vol. 33 309-317 (2012).

941 46. Bycroft, C. et al. The UK Biobank resource with deep phenotyping and genomic data.

$942 \quad$ Nature 562, 203-209 (2018).

943 47. Wang, K. et al. PennCNV: an integrated hidden Markov model designed for

944 high-resolution copy number variation detection in whole-genome SNP genotyping data.

945 Genome Res. 17, 1665-1674 (2007). 
48. Macé, A. et al. New quality measure for SNP array based CNV detection. Bioinformatics 32, 3298-3305 (2016).

49. Liaw, A. \& Wiener, M. Classification and Regression by Randomforest. $R$ news 2, 285

949 (2002).

950 50. Di Angelantonio, E. et al. Efficiency and safety of varying the frequency of whole blood 951 donation (INTERVAL): a randomised trial of 45000 donors. Lancet 390, 2360-2371 952 (2017).

953 51. Fromer, M. et al. Discovery and statistical genotyping of copy-number variation from 954 whole-exome sequencing depth. Am. J. Hum. Genet. 91, 597-607 (2012).

955 52. Backenroth, D. et al. CANOES: detecting rare copy number variants from whole exome 956 sequencing data. Nucleic Acids Res. 42, e97 (2014).

957 53. Packer, J. S. et al. CLAMMS: a scalable algorithm for calling common and rare copy 958 number variants from exome sequencing data. Bioinformatics 32, 133-135 (2016).

959 54. Crawford, K. et al. Medical consequences of pathogenic CNVs in adults: analysis of the 960 UK Biobank. J. Med. Genet. 56, 131-138 (2019).

961 55. McLaren, W. et al. The Ensembl Variant Effect Predictor. Genome Biology vol. 17 962 (2016).

963 56. Rentzsch, P., Witten, D., Cooper, G. M., Shendure, J. \& Kircher, M. CADD: predicting 964 the deleteriousness of variants throughout the human genome. Nucleic Acids Research 965 vol. 47 D886-D894 (2019).

966 57. Karczewski, K. J. et al. The mutational constraint spectrum quantified from variation in $967 \quad 141,456$ humans. Nature 581, 434-443 (2020).

968 58. Cummings, B. B. et al. Transcript expression-aware annotation improves rare variant 969 interpretation. Nature 581, 452-458 (2020).

970 59. Samocha, K. E. et al. Regional missense constraint improves variant deleteriousness 971 prediction. (2017) doi:10.1101/148353. 
972 60. Zhao, H. et al. CrossMap: a versatile tool for coordinate conversion between genome

973 assemblies. Bioinformatics 30, 1006-1007 (2014).

974 61. Kersey, P. J. et al. Ensembl Genomes 2016: more genomes, more complexity. Nucleic

$975 \quad$ Acids Res. 44, D574-80 (2016).

976 62. Jia, T., Munson, B., Lango Allen, H., Ideker, T. \& Majithia, A. R. Thousands of missing

977 variants in the UK Biobank are recoverable by genome realignment. Ann. Hum. Genet.

$978 \quad 84,214-220(2020)$.

979 63. Landrum, M. J. et al. ClinVar: improving access to variant interpretations and supporting 980 evidence. Nucleic Acids Res. 46, D1062-D1067 (2018).

981 64. Balduzzi, S., Rücker, G. \& Schwarzer, G. How to perform a meta-analysis with R: a 982 practical tutorial. Evid. Based. Ment. Health 22, 153-160 (2019).

983 65. Sweden, S. Multi-generation register 2016: A description of contents and quality.

984 (Statistics Sweden, Population and Welfare Department, 2017).

985 66. Carlstedt, B. Cognitive abilities - aspects of structure, process and measurement.

986 (University of Gothenburg, 2000).

987 67. Kolk, M. \& Barclay, K. Cognitive ability and fertility among Swedish men born

988 1951-1967: evidence from military conscription registers. Proc. Biol. Sci. 286, 20190359 989 (2019).

990 68. Hällsten, M. Inequality across three and four generations in Egalitarian Sweden: 1st and 991 2nd cousin correlations in socio-economic outcomes. Research in Social Stratification 992 and Mobility vol. 35 19-33 (2014).

993 69. Mårdberg, B. \& Carlstedt, B. Swedish Enlistment Battery (SEB): Construct Validity and 994 Latent Variable Estimation of Cognitive Abilities by the CAT-SEB. International Journal 995 of Selection and Assessment vol. 6 107-114 (1998).

996 70. Rönnlund, M., Carlstedt, B., Blomstedt, Y., Nilsson, L.-G. \& Weinehall, L. Secular trends 997 in cognitive test performance: Swedish conscript data 1970-1993. Intelligence vol. 41 
bioRxiv preprint doi: https://doi.org/10.1101/2020.05.26.116111; this version posted December 8, 2020. The copyright holder for this preprint (which was not certified by peer review) is the author/funder, who has granted bioRxiv a license to display the preprint in perpetuity. It is made available under aCC-BY 4.0 International license.

998

19-24 (2013).

999 